\title{
Hyper Chaotic Colpitts Oscillator for Wireless Power Transmission
}

\author{
Haixia Zhu ${ }^{1}$, Wei $\mathrm{Wei}^{2}$ and Wenbo $\mathrm{Liu}^{3, *}$ \\ ${ }^{1,2,3}$ College of Automatic Engineering, Nanjing University of Aeronautics and Astronautics, Nanjing, China \\ ${ }^{1}$ nanhang Jincheng College, Nanjing, China \\ *Corresponding author
}

\begin{abstract}
A new type of hyper chaotic oscillator is proposed based on the typical Colpitts chaotic oscillators. The chaotic signal is generated as the excitation signal of the wireless transmission system. Two types of Colpitts chaotic oscillation signals are compared from three aspects. We can find that the hyper chaotic Colpitts oscillation signal has the characteristics of more efficient wireless transmission efficiency compared to the typical Colpitts chaotic oscillation signal. Furthermore, the Complementary Cumulative Distribution Function curves show that the hyper chaotic Colpitts oscillation circuit has a preferable conversion efficiency of the rectifier circuit in the wireless power transmission system. Finally, the detailed chart of conversion efficiency of these circuits has been demonstrated.
\end{abstract}

Keywords-wireless power transmission; hyper chaotic oscillator; rectifier circuit

\section{INTRODUCTION}

In recent years, people's demand for electronic equipments has been greatly increased with the development of smart city. Wireless power transmission (WPT) technology has become one of the most rapidly growing, influential technologies, and will completely change the world. The structure diagram of the wireless transmission system is shown in Figure 1.

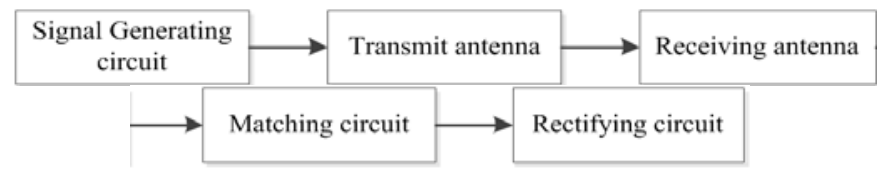

FIGURE I. THE STRUCTURE DIAGRAM OF THE WIRELESS TRANSMISSION SYSTEM

The chaotic signal has also been proved to improve the efficiency of wireless power transmission ${ }^{[1-3]}$. In this paper, we propose a new hyper chaotic circuit based on the typical Colpitts chaotic oscillator circuit ${ }^{[4]}$, which is proved to have better bandwidth and higher transmission efficiency in wireless transmission system.

\section{CHAOTIC COLPITTS OSCILlAter}

Chua's circuit is always the typical circuit of dynamic and chaotic characteristics of circuit system. While due to its spectrum width which is limited by the speed limit of the operational amplifier in the circuit, the frequency spectrum of the chaotic signal is more than $\mathrm{kHz}$ orders of magnitude. So far the highest experimental results reported in the literature are about $100 \mathrm{MHz}$ and the chaotic oscillation has been very unstable ${ }^{[5]}$. In 1994, the first time that Professor Kennedy found the Colpitts circuit can work in the chaos state ${ }^{[6]}$. Since then, the research on the Colpitts oscillation circuit is gradually expanded. The Colpitts circuit as an alternative for the design of the broadband chaotic oscillator becomes a new research hotspot.

The typical Colpitts chaotic oscillation circuit was shown in Figure 2. And the hyper chaotic Colpitts oscillation circuit was shown in Figure 3. Although the two circuits both use a triode transistor as a nonlinear device, the typical Colpitts chaotic oscillating circuit is a typical common base circuit, and the improved circuit is no longer a circuit with common nodes. More importantly, due to the parasitic capacitance $\mathrm{C}_{\mathrm{CB}}$ between the base and the collector (high frequency signal grounding), the chaotic oscillation frequency of the typical Colpitts chaotic oscillator is difficult to improve. When the circuit is improved, the inductance and resistance elements are added between the base of the transistor and the ground, the high frequency parasitic capacitance $\mathrm{C}_{\mathrm{CB}}$ is isolated, so that the chaotic oscillation of the circuit is not affected, and higher chaotic oscillation frequency can be obtained.

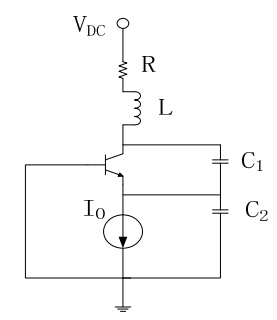

FIGURE II. THE TYPICAL COLPITTS CHAOTIC OSCILLATION CIRCUIT

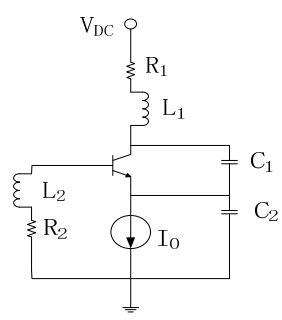

FIGURE III. THE HYPER CHAOTIC COLPITTS OSCILLATION CIRCUIT 


\section{A. ( $i$ ) State equations}

The state equations for the hyper chaotic oscillator circuit depicted in the Figure 3 are as:

$$
\left\{\begin{array}{l}
\mathrm{C}_{1} \frac{d \mathrm{C}_{\mathrm{c}_{1}}}{\mathrm{dt}}=\mathrm{I}_{\mathrm{L}_{1}}-\mathrm{f}\left(\mathrm{V}_{\mathrm{BE}}\right)+\mathrm{I}_{\mathrm{L}_{2}} \\
\mathrm{C}_{2} \frac{\mathrm{dV}_{\mathrm{c}_{2}}}{\mathrm{dt}}=\mathrm{I}_{\mathrm{L}_{1}}+\mathrm{I}_{\mathrm{L}_{2}}-\mathrm{I}_{0} \\
\mathrm{~L}_{1} \frac{\mathrm{dI} \mathrm{L}_{\mathrm{L}_{1}}}{\mathrm{dt}}=\mathrm{V}_{\mathrm{DC}}-\mathrm{I}_{\mathrm{L}_{1}} \mathrm{R}_{1}-\mathrm{V}_{\mathrm{C}_{1}}-\mathrm{V}_{\mathrm{C}_{2}} \\
\mathrm{~L}_{2} \frac{\mathrm{dI} \mathrm{L}_{\mathrm{L}_{2}}}{\mathrm{dt}}=-\mathrm{I}_{\mathrm{L}_{2}} \mathrm{R}_{4}-\mathrm{V}_{\mathrm{BE}}-\mathrm{V}_{\mathrm{C}_{2}}
\end{array}\right.
$$

And the state equations can be changed as follows:

$$
\begin{aligned}
& \int \mathrm{C}_{1} \frac{\mathrm{dV} \mathrm{C}_{\mathrm{c}_{1}}}{\mathrm{dt}}=\mathrm{I}_{\mathrm{L}_{1}}-\mathrm{f}\left(\mathrm{V}_{\mathrm{BE}}\right)+\mathrm{I}_{\mathrm{L}_{2}} \\
& \mathrm{C}_{2} \frac{\mathrm{dV}_{\mathrm{c}_{2}}}{\mathrm{dt}}=\mathrm{I}_{\mathrm{L}_{1}}+\mathrm{I}_{\mathrm{L}_{2}}-\mathrm{I}_{0} \\
& \mathrm{~L}_{1} \frac{\mathrm{dI}_{\mathrm{L}_{1}}}{\mathrm{dt}}=\mathrm{V}_{\mathrm{DC}}-\mathrm{I}_{\mathrm{L}_{1}} \mathrm{R}_{1}-\mathrm{V}_{\mathrm{C}_{1}}-\mathrm{V}_{\mathrm{C}_{2}} \\
& \mathrm{~L}_{2} \frac{\mathrm{dI}_{\mathrm{L}_{2}}}{\mathrm{dt}}=-\mathrm{I}_{\mathrm{L}_{2}} \mathrm{R}_{4}-\mathrm{V}_{\mathrm{BE}}-\mathrm{V}_{\mathrm{C}_{2}} \\
& \mathrm{C}_{1} \frac{\mathrm{dV} \mathrm{BE}_{\mathrm{BE}}}{\mathrm{dt}}=\mathrm{I}_{\mathrm{L}_{1}}-\mathrm{f}\left(\mathrm{V}_{\mathrm{BE}}\right)+\mathrm{I}_{\mathrm{L}_{2}}+\frac{\mathrm{C}_{1}}{\mathrm{C}_{\mathrm{CB}}}\left(\mathrm{I}_{\mathrm{L}_{2}}-\mathrm{I}_{\mathrm{b}}\right)
\end{aligned}
$$

Among the state equations:

$$
\begin{aligned}
& f\left(V_{\mathrm{BE}}\right)= \begin{cases}\mathrm{I}_{0} \frac{V_{\mathrm{BE}}-V_{\mathrm{TH}}}{V_{\mathrm{T}}}, & V_{\mathrm{BE}}>V_{\mathrm{TH}}, V_{\mathrm{T}}=26 \mathrm{mV}, \\
0 \quad, V_{\mathrm{BE}} \leq V_{\mathrm{TH}}\end{cases} \\
& \mathrm{V}_{\mathrm{TH}}=\mathrm{V}_{\mathrm{T}} \log \frac{\mathrm{I}_{0}}{\mathrm{I}_{\mathrm{S}}}
\end{aligned}
$$

\section{B. (ii) Simulation}

The chaotic oscillation circuit was fabricated using the BFP183W transistor. In the actual circuit design, the price of the current source is more expensive, so we use a resistance and a negative DC voltage source to replace it. The selected components parameter values in Figure 2: $\mathrm{L}=18 \mathrm{nH}$, $\mathrm{C}_{1}=10 \mathrm{pF}, \mathrm{C}_{2}=10 \mathrm{pF}, \mathrm{R}=26 \Omega, \mathrm{V}_{\mathrm{DC}}=7 \mathrm{~V}$. The selected components parameter values in Figure 3: $\mathrm{L}_{1}=8 \mathrm{nH}, \mathrm{L}_{2}=12 \mathrm{nH}, \mathrm{C}_{1}=8 \mathrm{pF}, \mathrm{C}_{2}=10 \mathrm{pF}, \mathrm{R}_{1}=26 \Omega, \mathrm{R}_{2}=10 \Omega, \mathrm{V}_{\mathrm{DC}}=7 \mathrm{~V}$. Time domain diagram, phase diagram and spectrum diagram of typical Colpitts chaotic signal are shown in Figure 4. Time domain diagram, phase diagram and spectrum diagram of the hyper chaotic Colpitts signal are shown in Figure 5.

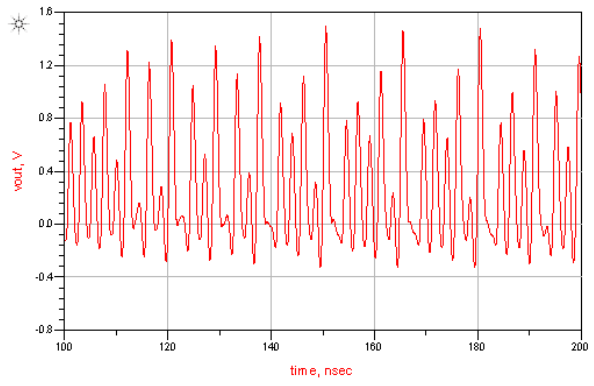

(a) Time domain diagram

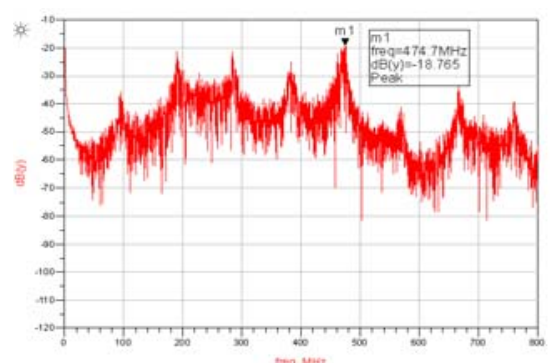

(b) Spectrum diagram

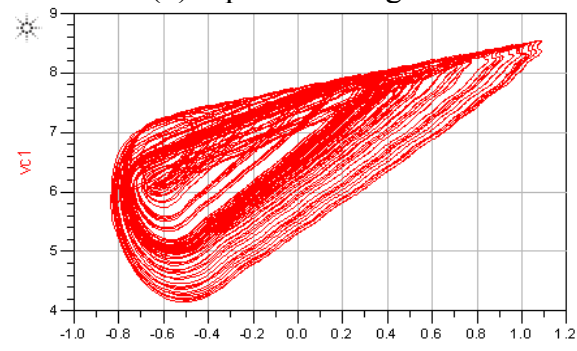

ve2

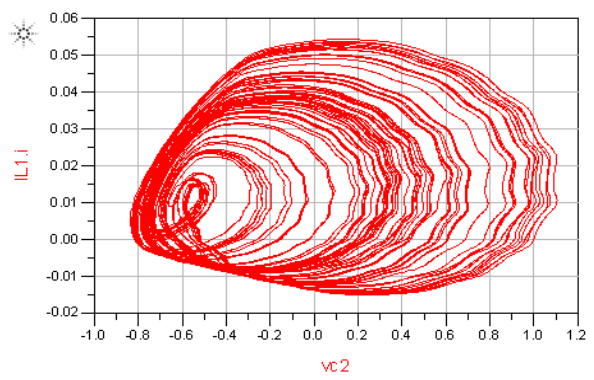

(c) Phase diagram

FIGURE IV. THE TIME DOMAIN DIAGRAM(A), SPECTRUM DIAGRAM (B) AND PHASE DIAGRAM (C) OF TYPICAL COLPITTS CHAOTIC SIGNAL

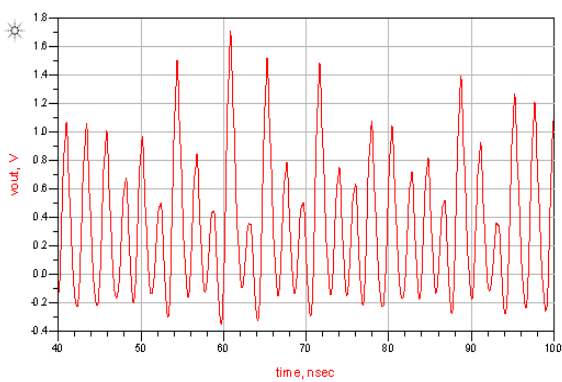

(a) Time domain diagram

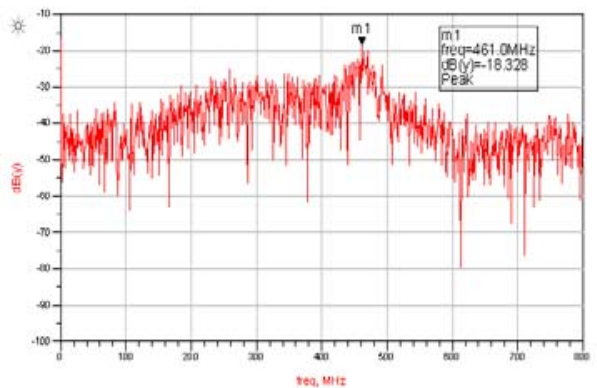

(b) Spectrum diagram 

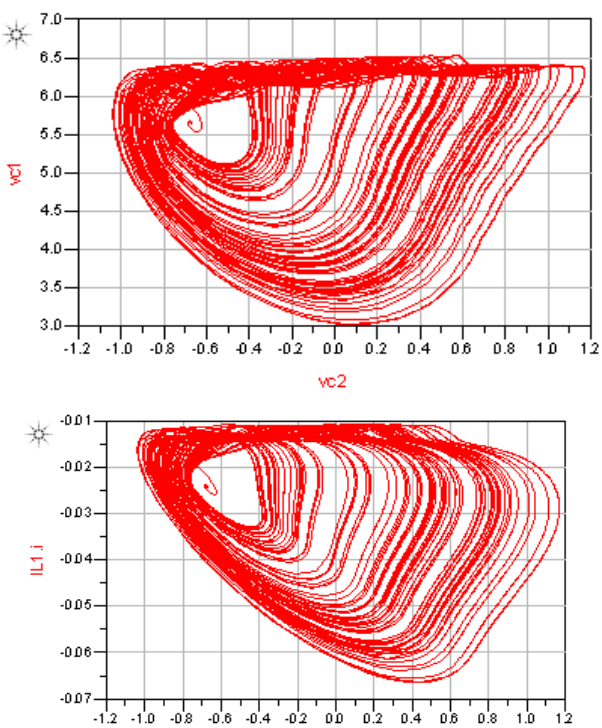

$w 2$
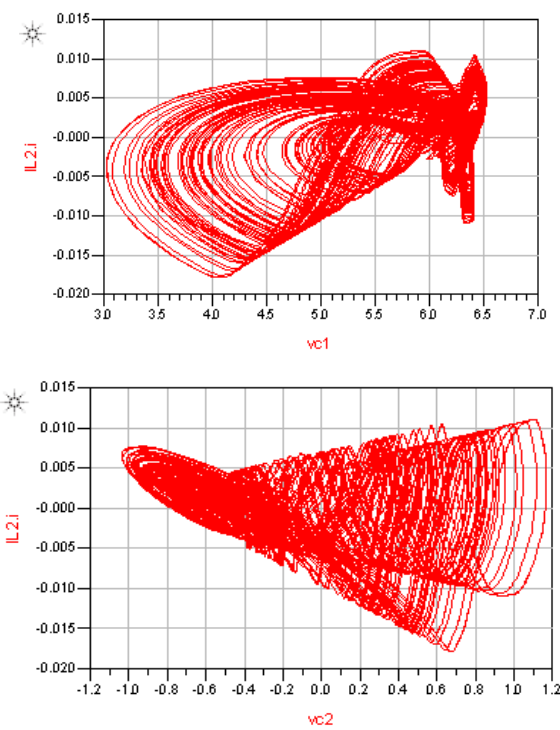

(c) Phase diagram

FIGURE V. THE TIME DOMAIN DIAGRAM (A), SPECTRUM DIAGRAM (B) AND PHASE DIAGRAM (C) OF HYPER CHAOTIC COLPITTS SIGNAL

From the Figure 4 (a) and Figure 5 (a), We can see the waveforms generated by the two circuits appears to have no rules, while with the passage of time, the voltage amplitude of the output signals both have been in a certain range of random. The phase diagrams of the signals are all typical chaotic attractors. Because the circuit in Figure 3 is more than three-dimensional, the phase diagrams of it can demonstrate that the signal generated by it is a hyper chaotic signal. By comparison of the spectrum diagram, the $\mathrm{dB}$ value of the center frequency of the typical Colpitts chaotic oscillation signal is $-18.765 \mathrm{~dB}$. While there are several small spikes besides the center frequency of the typical Colpitts chaotic oscillation signal, which makes the frequency spectrum of the whole is lower, and is not conducive to improving the efficiency of wireless power transmission. The $\mathrm{dB}$ value of the hyper chaotic Colpitts oscillator signal is $-18.328 \mathrm{~dB}$, nearly the same with the typical Colpitts chaotic oscillation signal at the center frequency. Also the value around the center frequency drops slowly and the bandwidth is very wide. From $200 \mathrm{MHz}$ to $540 \mathrm{MHZ}$, the dB value is above $-40 \mathrm{~dB}$, which is beneficial to improve the efficiency of the wireless power transmission.

\section{RECTIFIER CIRCUIT DESIGN}

In order to prove that the chaotic signal can improve the electromagnetic energy collection and the efficiency of the wireless power transmission, the rectifier circuit ${ }^{[8]}$ required for the experimental is shown in Figure 6. The excitation signal source $v_{\text {out }}$ generated by the chaotic oscillation circuit was transmitted by a transmit antenna and received by the receiving antenna to the rectifying circuit. By means of a T type matching network, which is necessary to provide maximum power transfer from antenna to the rectifier, the rectifier circuit is matched to $50 \mathrm{ohm}$ load. The RF to DC conversion efficiency of the rectifier is optimized in order to maximize the harmonic balance simulation of approximately $450 \mathrm{MHz}$.

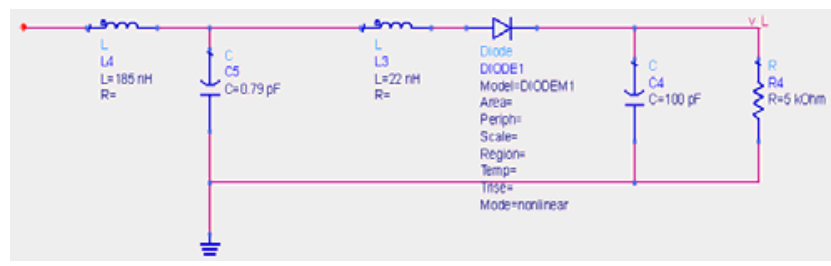

FIGURE VI. THE RECTIFIER CIRCUIT

\section{COMPARISOn Of ReCTIFIER PERFormance}

In this paper, the chaotic signals generated by the typical Colpitts chaotic oscillation circuit and the hyper chaotic oscillation circuit are used as the excitation signal of the wireless transmission system, so as to prove the effectiveness of the chaotic signal to improve the efficiency of wireless power transmission.

The PAPR $(\mathrm{dB})$ of a waveform can be defined as:

$$
P A P R=10 \log _{10} \frac{\max \left[x(t)^{2}\right]}{\left\langle x(t)^{2}\right\rangle}
$$

where $x(t)$ is the time domain waveform of the single and \langle\rangle refers to the time average operater.

The PAPR can also be defined with respect to the envelope of the single $e(t)$ :

$$
\operatorname{PAPR}(x(t))=\operatorname{PAPR}(e(t))+3 d B
$$

The PCCDF curve indicates the probability that the PAPR of the envelope signal exceeds a certain threshold. The maximum PAPR of the envelope signal in the pCCDF curve is the maximum. The maximum PAPR of the signal can be obtained 
by increasing $3 \mathrm{~dB}$ to the maximum PAPR of the envelope signal.

The power CCDF (typically called pCCDF) measurement is a very common measurement of the conversion efficiency in rectifier circuit ${ }^{[4]}{ }^{[13]}$. The CCDF curve shows the probability that the instantaneous signal power will be higher than the average signal power by a certain amount of $d B$. The independent axis of the CCDF curve shows power levels in $\mathrm{dB}$ with respect to the signal average power level $(0 \mathrm{~dB}$ corresponds to the signal average power level). The dependent axis of the CCDF curve shows the probability that the instantaneous signal power will exceed the corresponding power level on the independent axis. In Figure 7(a), the pCCDF curve is for the typical Colpitts chaotic signal and the pCCDF1 curve is for the hyper chaotic Colpitts signal.

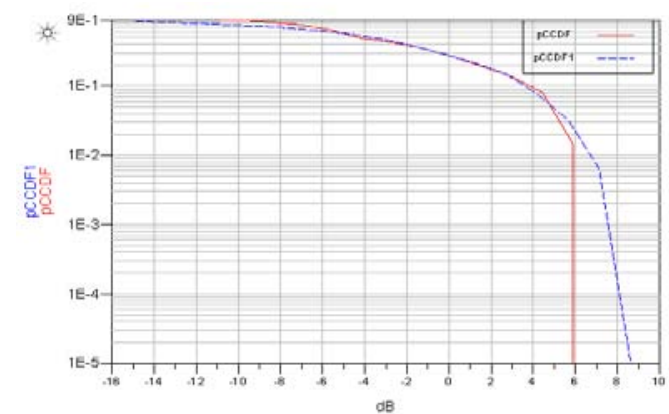

(a)

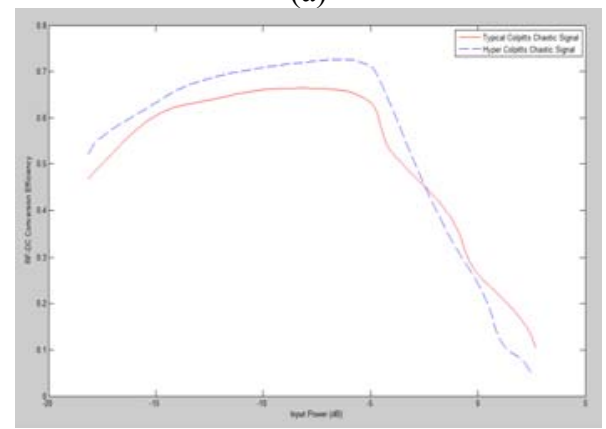

(b)

FIGURE VII. (A) PCCDF CURVES OF TYPICAL COLPITTS CHAOTIC SIGNAL AND THE HYPER CHAOTIC COLPITTS SIGNAL, (B) RF-DC CONVERSION EFFICIENCY OF THE RECTIFIER CIRCUIT

By the pCCDF curves, before $4 \mathrm{~dB}$, the pCCDF value of the typical Colpitts chaotic signal is almost the same with that of the hyper chaotic signal. While after $4 \mathrm{~dB}$, the pCCDF value of the typical Colpitts chaotic signal drops down and is lower than that of the hyper chaotic signal. When the pCCDF value equals $1 \mathrm{E}-4$, the $\mathrm{dB}$ value of the hyper chaotic Colpitts signal is $8 \mathrm{~dB}$ while that of the typical Colpitts chaotic signal is only $6 \mathrm{~dB}$. So the PAPR value of the typical Colpitts chaotic signal is $9 \mathrm{~dB}$, and the PAPR value of the hyper chaotic Colpitts signal is $11 \mathrm{~dB}$. The experimental results show that the PAPR of the typical chaotic signal is lower than that of the hyper chaotic signal.

The collection of electromagnetic energy is converted to the conversion efficiency, which is based on the received radio frequency signal. The RF-DC conversion efficiency was evaluated using (5).

$$
\eta=\frac{p_{\text {gut }}}{P_{\text {in }}}=\frac{v_{\text {gut }}^{\mathrm{N}} / \mathbb{R}_{\mathrm{n}}}{P_{\text {in. }}}
$$

Figure 7(b) compares the conversion efficiency of the typical Colpitts chaotic signal and the hyper chaotic Colpitts signal with different input power. From the Figure 7(b), we can see that before $-2 \mathrm{~dB}$, the hyper chaotic signal has a higher conversion efficiency compared with the typical chaotic signal. While after $-2 \mathrm{~dB}$, the conversion efficiency of the hyper chaotic signal is lower.

\section{CONCLUSION}

We analyzed the chaotic oscillation signals in three aspects: time domain diagram, phase diagram and spectrum diagram. In addition, we compare the complementary cumulative distribution function curves and the conversion efficiency curves of two types of Colpitts chaotic oscillation signals. The preferable conversion efficiency at low input power of the hyper Colpitts oscillating circuit is demonstrated by experiments.

\section{REFERENCES}

[1] Lo C C, et al, Novel wireless impulsive power transmission to enhance the conversion efficiency for low input power, Microwave Workshop Series on Innovative Wireless Power Transmission: Technologies, Systems, and Applications (IMWS),IEEE, (2011) 55-58.

[2] Abdullah H N, et al. Efficient chaotic communication system for wireless sensing applications, Systems, Signals and Devices (SSD), 2012 9th International Multi-Conference on. IEEE, (2012)1-5.

[3] Collado A, et al, Optimal Waveforms for Efficient Wireless Power Transmission. (2014).

[4] Yang Y L, et al, Efficiency improvement of the impulsive wireless power transmission through biomedical tissues by varying the duty cycle, Microwave Workshop Series on Innovative Wireless Power Transmission: Technologies, Systems, and Applications (IMWS),IEEE, (2011) 175-178.

[5] Datcu O, et al, Experimental impulsive observer with colpitts oscillators, Signals, Circuits and Systems (ISSCS), 2013 International Symposium on. IEEE, (2013)1-4.

[6] Jingxia L, Fuchang M, Chaos Generation in Microwave Band Using Improved Colpitts Oscillator[C], IEEE Computer Society, (2012)405-408.

[7] Collado A, et al,Improving wireless power transmission efficiency using chaotic waveforms[C], Microwave Symposium Digest (MTT), 2012 IEEE MTT-S International. IEEE, (2012) 1-3.

[8] Boaventura A, Belo D, Fernandes R, et al, Boosting the Efficiency: Unconventional Waveform Design for Efficient Wireless Power Transfer, IEEE Microwave Magazine. 16 (2015) 87-96.

[9] Megherbi O, et al, A novel transmission scheme based on impulsive synchronization of two Colpitts chaotic systems, Systems and Control (ICSC), 2013 3rd International Conference on. IEEE, ( 2013) 117-122.

[10] Chen W, et al, A novel ultra-wideband microwave chaotic Colpitts oscillator, Wireless and Microwave Technology Conference (WAMICON), 2013 IEEE 14th Annual. IEEE, (2013)1-4.

[11] Susan R J, et al, Chaotic sequences for reduction of PAPR in OFDMA system[C], India Conference (INDICON), 2011 Annual IEEE. IEEE,(2011) 1-4.

[12] Chung $\mathrm{C}$, et al, Robust Colpitts and Hartley oscillator design[C], Frequency Control Symposium (FCS), 2014 IEEE International. IEEE. (2014)1-6.

[13] Ha K W, et al, -Boosted Complementary Current-Reuse Colpitts VCO With Low Power and Low Phase Noise[J], IEEE Microwave \& Wireless Components Letters. 24 (2014) 418-420. 